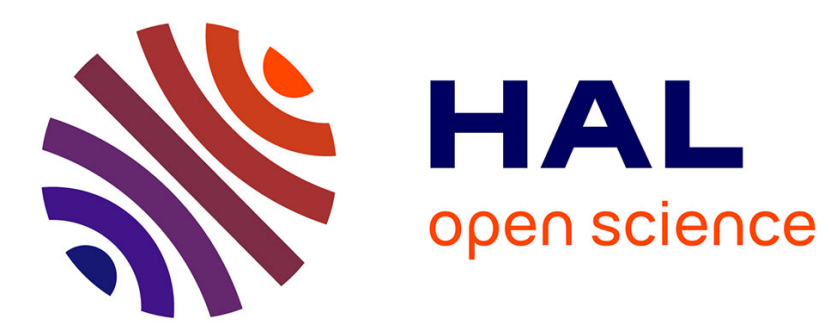

\title{
On the problem of instability in the dimension of a spline space over a T-mesh
}

Berdinsky Dmitry, Oh Min-Jae, Kim Taewan, Bernard Mourrain

\section{To cite this version:}

Berdinsky Dmitry, Oh Min-Jae, Kim Taewan, Bernard Mourrain. On the problem of instability in the dimension of a spline space over a T-mesh. Computers and Graphics, 2012, 36 (5), pp.507-513. 10.1016/j.cag.2012.03.005 . hal-00742505

\section{HAL Id: hal-00742505 \\ https://hal.inria.fr/hal-00742505}

Submitted on 16 Oct 2012

HAL is a multi-disciplinary open access archive for the deposit and dissemination of scientific research documents, whether they are published or not. The documents may come from teaching and research institutions in France or abroad, or from public or private research centers.
L'archive ouverte pluridisciplinaire $\mathbf{H A L}$, est destinée au dépôt et à la diffusion de documents scientifiques de niveau recherche, publiés ou non, émanant des établissements d'enseignement et de recherche français ou étrangers, des laboratoires publics ou privés. 


\title{
On the problem of instability in the dimension of a spline space over a T-mesh
}

\author{
Dmitry Berdinsky ${ }^{\mathrm{a}}$, Min-jae $\mathrm{Oh}^{\mathrm{a}}$, Tae-wan Kim ${ }^{\mathrm{a}, \mathrm{b}, *}$, Bernard Mourrain ${ }^{\mathrm{c}}$ \\ ${ }^{a}$ Department of Naval Architecture and Ocean Engineering, Seoul National University, Seoul 151-744, Republic of Korea \\ ${ }^{b}$ Research Institute of Marine Systems Engineering, Seoul National University, Seoul 151-744, Republic of Korea \\ ${ }^{c}$ GALAAD, INRIA Méditerranée, BP 93, 06902 Sophia Antipolis, France
}

\begin{abstract}
In this paper, we discuss the problem of instability in the dimension of a spline space over a T-mesh. For bivariate spline spaces $S(5,5,3,3)$ and $S(4,4,2,2)$, the instability in the dimension is shown over certain types of T-meshes. This result could be considered as an attempt to answer the question of how large the polynomial degree $\left(m, m^{\prime}\right)$ should be relative to the smoothness $\left(r, r^{\prime}\right)$ to make the dimension of a spline space stable. We show in particular that the bound $m \geq 2 r+1$ and $m^{\prime} \geq 2 r^{\prime}+1$ are optimal.
\end{abstract}

Keywords:

Spline space, T-mesh, Dimension

\section{Introduction}

This paper is motivated by a recent work [1] devoted to the observation of instability in the dimension of a spline space $S(m, m, m-1, m-1)$ over a T-mesh.

Splines over a T-mesh [2] could be a useful tool in many areas such as surface modeling and finite element analysis. For instance, polynomial splines over hierarchical T-meshes (PHT-splines) [3] appear to be a natural generalization of B-splines over hierarchical Tmeshes. A method has been proposed for stitching several surface patches and for fitting genus-zero meshes using PHT-splines [4]. Splines over T-meshes allow one to avoid the problem of superfluous control points, because inserting an extra node implies only local refinement of a control mesh. From this point of view, splines over T-mesh also have a good prospect for using in isogeometric analysis [5]; for instance, see [6], [7].

However, unfortunately, the problem of describing the basis functions of a spline space over a given Tmesh is generally non-trivial. For bicubic splines with continuity of order one, an efficient algorithm has been proposed to construct basis functions over a general Tmesh [8]; these basis functions satisfy remarkable properties such as local support, nonnegativity, and partition of unity. However, for bicubic splines with continuity of

\footnotetext{
${ }^{*}$ Corresponding author

Email address: taewan@snu.ac.kr (Tae-wan Kim)

URL: http://caditlab.snu.ac.kr (Tae-wan Kim)
}

order two, even the dimension is not easy to calculate, and it is exactly known only for some particular classes of T-meshes. Therefore, the dimension is one of the crucial characteristics of a spline space over a T-mesh that must be analyzed.

We briefly recall some basic notations and recent results concerning the dimension of a spline space over a T-mesh.

A T-mesh is basically a subdivision of some domain into rectangular cells that allow T-junctions (see Fig. 1). In this paper, only a rectangular domain is considered.

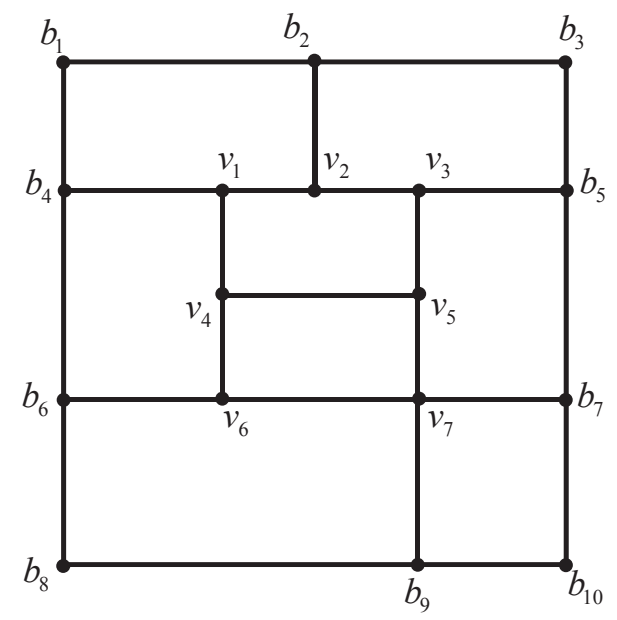

Figure 1: Example of a T-mesh. 
Let $S\left(m, m^{\prime}, r, r^{\prime}\right)(T)$ denote the space of functions of variables $x, y$ that are piecewise polynomials of degree $m, m^{\prime}$ and the class of smoothness $C^{r, r^{\prime}}$ over the given Tmesh $T$. We also refer to $S\left(m, m^{\prime}, r, r^{\prime}\right)(T)$ as the spline space over T-mesh $T$.

In [2], the exact formula for the dimension of the space $S\left(m, m^{\prime}, r, r^{\prime}\right)(T)$ is obtained when $m \geq 2 r+1$, $m^{\prime} \geq 2 r^{\prime}+1$. In this case,

$$
\begin{array}{r}
\operatorname{dim} S\left(m, m^{\prime}, r, r^{\prime}\right)(T)=(m+1)\left(m^{\prime}+1\right) f_{2}- \\
(m+1)\left(r^{\prime}+1\right) f_{1}^{h}-\left(m^{\prime}+1\right)(r+1) f_{1}^{v}+ \\
(r+1)\left(r^{\prime}+1\right) f_{0},
\end{array}
$$

where $f_{2}$ is the number of cells of $T, f_{1}^{h}$ and $f_{1}^{v}$ respectively are the numbers of horizontal and vertical interior edges (these intersect the interior of the rectangular domain of $T$ ), and $f_{0}$ is the number of interior vertices (these are in the interior of the rectangular domain). For instance, in Fig. $1, v_{1}, \ldots, v_{7}$ are interior vertices; $b_{2} v_{2}, v_{1} v_{4}$, vertical interior edges; and $b_{4} v_{1}, v_{6} v_{7}$, horizontal interior edges.

The method used to calculate the dimension of $S\left(m, m^{\prime}, r, r^{\prime}\right)(T)$ in [2] is based on Bézier nets. In other words, in [2], the formula for the number of elements in a minimal determining set of Bézier ordinates is obtained.

Proofs of analogous results have been presented in [9] and [10] independently. However, the approach used in these studies is based on the smoothing cofactor method.

All these methods are based on the following simple observation. Let $P_{1}(x, y)$ and $P_{2}(x, y)$ be two polynomials of degree $m, m^{\prime}$ defined on rectangular domains $\Omega_{1}$ and $\Omega_{2}$, respectively, and having the common interval $\left(v_{1}, v_{2}\right)$ (see Fig. 2). Then, the piecewise polynomial defined on $\Omega_{1} \cup \Omega_{2}$ has $r$-th order of smoothness for variable $x$, if and only if

$$
P_{1}(x, y)-P_{2}(x, y)=\lambda(x, y)\left(x-x_{0}\right)^{r+1},
$$

for some polynomial $\lambda(x, y)$ of degree $m-r-1, m^{\prime}$ for variables $x, y$, respectively.

Here, we do not provide any detailed explanations of the approach used in these studies, but only mention the fact that the smoothing cofactor method appears to be the basic method for all further results concerning the dimension of a spline space over a T-mesh.

Thus, when $m \geq 2 r+1, m^{\prime} \geq 2 r^{\prime}+1$, one can express the dimension formula in simple topological quantities such as the number of cells, edges, and vertices. This fact implies that any small variation, saving the topology of T-mesh $T$, does not change the dimension of the spline space $S\left(m, m^{\prime}, r, r^{\prime}\right)(T)$.

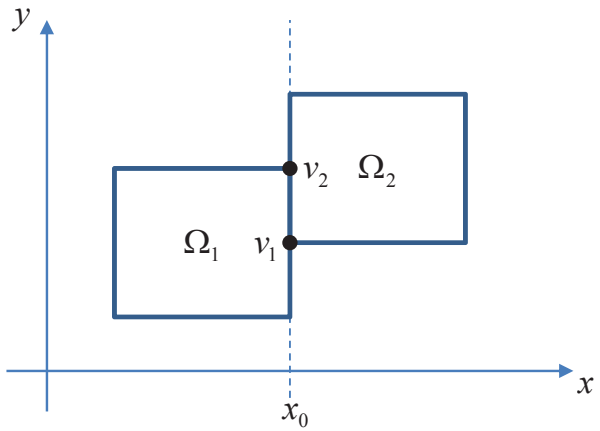

Figure 2: Two adjacent cells.

We will show hereafter that the conditions $m \geq 2 r+1$, $m^{\prime} \geq 2 r^{\prime}+1$ are optimal by giving explicit cases where $m=2 r, m^{\prime}=2 r^{\prime}$ or $m=2 r-1, m^{\prime}=2 r^{\prime}-1$ for which the dimension of the spline space changes with the position of the vertices.

In [10], Li et al. made the following improvement to the dimension formula. They found the constraint on the T-mesh that guarantees the dimension of the corresponding spline space to be calculated using formula (1).

It appears that the last progress in the analysis of the dimension of a spline space, in a general case, was made in [11]. In this study, the results presented in [9] and [10] were reproved. In addition to the smoothing cofactor method, [11] exploited homology techniques to provide additional insight on the analysis of the dimension of the spline spaces. Finally, the dimension of a space $S\left(m, m^{\prime}, r, r^{\prime}\right)(T)$ is represented in this study in the following manner:

$$
\begin{array}{r}
\operatorname{dim} S\left(m, m^{\prime}, r, r^{\prime}\right)(T)=(m+1)\left(m^{\prime}+1\right) f_{2}- \\
(m+1)\left(r^{\prime}+1\right) f_{1}^{h}-\left(m^{\prime}+1\right)(r+1) f_{1}^{v}+ \\
(r+1)\left(r^{\prime}+1\right) f_{0}+h_{m, m^{\prime}}^{r, r^{\prime}}(T),
\end{array}
$$

where the meaning of the term $h_{m, m^{\prime}}^{r, r^{\prime}}(T)$ is described in the next section.

Recall that the hierarchical T-mesh is either the initial square or is obtained from a hierarchical T-mesh by splitting a cell along a vertical or horizontal line. A valuable observation was made in [11] about the upper estimation of the summand $h_{m, m^{\prime}}^{r, r^{\prime}}(T)$ for a hierarchical T-mesh $T$. This estimation allows one to establish the constraint on a hierarchical T-mesh $T$ for the summand $h_{m, m^{\prime}}^{r, r^{\prime}}(T)$ to be zero, and this constraint, in some manner, generalizes the one obtained in [10]. Therefore, if a hierarchical T-mesh $T$ satisfies the constraint 
proposed in [11], then the dimension of a spline space $S\left(m, m^{\prime}, r, r^{\prime}\right)(T)$ is given by $(1)$.

In a general case, the formula for the dimension of a space $S\left(m, m^{\prime}, r, r^{\prime}\right)(T)$ cannot be expressed using only the simple topological characteristics of $T$, such as $f_{2}, f_{1}^{h}, f_{1}^{v}$, and $f_{0}$.

Recently, Li and Chen [1] demonstrated that the dimension of a space $S(m, m, m-1, m-1)(T)$ may also depend on the geometry of a T-mesh $T$.

For instance, the dimension of a space $S(3,3,2,2)(T)$ for $T$, shown in Fig. 3, depends on the values of the coordinates $s_{i}, t_{i}, i=1 \ldots 6$. There are certain values of the coordinates $s_{i}, t_{i}, i=1 \ldots 6$ for which the dimension jumps with a small variation in these values; this indicates the instability in the dimension of space $S(3,3,2,2)(T)$.

The interesting question stated in [1] is how large the polynomial degree should be relative to the smoothness for the dimension of a spline space to be stable. In this work, we treat the new cases, $S(4,4,2,2)(T)$ and $S(5,5,3,3)(T)$, and provide certain examples of Tmeshes $T$ with instability in the dimension of the spline space. This shows that the constraints $m \geq 2 r+1$, $m^{\prime} \geq 2 r^{\prime}+1$ known to yield a combinatorial dimension formula are optimal.

We note that the key approach to construct examples of T-meshes with instability in the dimension was in using the abovementioned constraint on a T-mesh. The key was to violate this constraint to guarantee that the dimension is not given by (1). Unfortunately, the summand $h_{m, m^{\prime}}^{r, r^{\prime}}(T)$ for some T-mesh $T$ should be calculated as a rank of the corresponding matrix. However, it is difficult to control even for small degrees $m, m^{\prime}$. Therefore, we specified coordinates of some nodes to simplify the calculations. As shown in the next section, the number of rows of this matrix linearly depends on the number of maximal interior segments. Therefore, to minimize the matrix size and make its rank unstable, we treated T-meshes with four maximal interior segments.

We investigated the cases $S(4,4,2,2)(T)$ and $S(5,5,3,3)(T)$ because after the result of [1], they were the first natural candidates to test the stability in the dimension. The other motivation was that splines with small polynomial degree could be of interest in many applications.

We also assume that further generalizations of our results require more sophisticated techniques. We believe that investigating the instability in the dimension of a space $S\left(m, m^{\prime}, r, r^{\prime}\right)(T)$ is an important issue because it provides a better understanding of the main problem, namely, how the basis of a space $S\left(m, m^{\prime}, r, r^{\prime}\right)(T)$ can be described.
The remainder of this work is organized as follows. In section 2, we explain the dimension formula (2) in detail and interpret it in a form pertinent for showing the instability in the dimension. In section 3 , we explain how the dimension formula (2) can be used to prove the instability in the dimension of a spline space $S(3,3,2,2)(T)$ for certain types of T-meshes $T$ described in [1]. In section 4, we describe certain types of T-meshes $T$ and prove the instability in the dimension of spline spaces $S(5,5,3,3)(T)$ and $S(4,4,2,2)(T)$. In section 5 , we present the conclusions of this work and discuss future works.

\section{Dimension of a spline space $S\left(m, m^{\prime}, r, r^{\prime}\right)(T)$ over T-mesh $T$ : General formula}

In this section, we explain the dimension formula (2) in detail. We also transform it into a form pertinent for showing the instability in the dimension. For reference, one can also find similar types of formulas for the dimension of a spline space in previous works [9],[10].

The general formula (2) for the dimension of the space $S\left(m, m^{\prime}, r, r^{\prime}\right)(T)$ for some T-mesh $T$ of the rectangular domain is found by using the homology technique [11].

The summand $h_{m, m^{\prime}}^{r, r^{\prime}}(T)$ in (2) may be considered as the dimension of the factor space $M / K$ [11]. The space $M$ is the direct sum

$$
M=\bigoplus_{\rho \in M I S(T)}[\rho] R_{\left(m, m^{\prime}\right)-\delta(\rho)}
$$

over the entire set of maximal interior segments $\operatorname{MIS}(T)$.

The maximal interior segment is the maximal union of connected edges of the same direction, such that there is no intersection with the border of the domain (for example, the segments $v_{1} v_{6}$ and $v_{4} v_{5}$ are all maximal interior segments of the T-mesh shown in Fig. 1).

Here, $R_{\left(m, m^{\prime}\right)}$ denotes the space of polynomials of degree $m, m^{\prime}$ of variables $x, y$, respectively, and $\delta(\rho)=$ $\left(0, r^{\prime}+1\right)$ if $\rho$ is the horizontal segment and $\delta(\rho)=$ $(r+1,0)$ if $\rho$ is the vertical segment.

One can consider the space $M$ as simply a vector space formed by the formal sums $\Sigma_{\rho \in M I S(T)} \beta_{\rho}[\rho]$, where $\beta_{\rho} \in R_{\left(m, m^{\prime}-r^{\prime}-1\right)}$ if $\rho$ is the horizontal maximal interior segment, and $\beta_{\rho} \in R_{\left(m-r-1, m^{\prime}\right)}$ if $\rho$ is the vertical maximal interior segment.

The subspace $K \subset M$ has the following form:

$$
K=\Sigma_{\gamma \in T_{0}^{0}}\left(\Delta_{h}^{r, r^{\prime}}\left[\rho_{v}(\gamma)\right]-\Delta_{v}^{r, r^{\prime}}\left[\rho_{h}(\gamma)\right]\right) R_{\left(m-r-1, m^{\prime}-r^{\prime}-1\right)},
$$


where $T_{0}^{0}$ is the set of vertices of maximal interior segments (for instance $v_{1}, v_{4}, v_{5}$, and $v_{6}$ are all points of $T_{0}^{0}$ for the T-mesh shown in Fig. 1). In addition,

$$
\Delta_{h}^{r, r^{\prime}}=\left(y-y_{\gamma}\right)^{r^{\prime}+1}, \Delta_{v}^{r, r^{\prime}}=\left(x-x_{\gamma}\right)^{r+1},
$$

where $x_{\gamma}, y_{\gamma}$ denotes the $x, y$ coordinates of the vertex $\gamma$. Furthermore, $\rho_{h}(\gamma)$ and $\rho_{v}(\gamma)$ are the corresponding horizontal and vertical maximal interior segments containing the vertex $\gamma$ (for example, in Fig. 1, $\rho_{h}\left(v_{4}\right)=$ $\left.v_{4} v_{5}, \rho_{v}\left(v_{4}\right)=v_{1} v_{6}\right)$. For more details, see [11].

To investigate the question of instability in the dimension of a space $S\left(m, m^{\prime}, r, r^{\prime}\right)(T)$ over some T-mesh $T$, it is only necessary to consider the summand $h_{m, m^{\prime}}^{r, r^{\prime}}(T)$.

Let $n$ be the number of vertices of $T_{0}^{0}$, and $l_{h}, l_{v}$ be the numbers of horizontal and vertical maximal interior segments, respectively. One can interpret $h_{m, m^{\prime}}^{r, r^{\prime}}(T)$ for some T-mesh $T$ in terms of the rank of the corresponding matrix. Indeed,

$$
\begin{array}{r}
h_{m, m^{\prime}}^{r, r^{\prime}}=\operatorname{dim} M-\operatorname{dim} K=(m+1)\left(m^{\prime}-r^{\prime}\right) l_{h}+ \\
\left(m^{\prime}+1\right)(m-r) l_{v}-(m-r)\left(m^{\prime}-r^{\prime}\right) n+
\end{array}
$$$$
\operatorname{dim} N \text {. }
$$

where $N$ is the subset

$$
N \subset \bigoplus_{\gamma \in T_{0}^{0}} R_{\left(m-r-1, m^{\prime}-r^{\prime}-1\right)},
$$

so that each element of $N$ is a set of polynomials

$$
\left\langle\alpha_{\gamma} \in R_{\left(m-r-1, m^{\prime}-r^{\prime}-1\right)}, \gamma \in T_{0}^{0}\right\rangle,
$$

such that it satisfies the following conditions:

$$
\Sigma_{\gamma \in \rho_{h}} \alpha_{\gamma}\left(x-x_{\gamma}\right)^{r+1}=0
$$

for each horizontal maximal interior segment $\rho_{h}$, and

$$
\Sigma_{\gamma \in \rho_{\nu}} \alpha_{\gamma}\left(y-y_{\gamma}\right)^{r^{\prime}+1}=0
$$

for each vertical maximal interior segment $\rho_{v}$.

However, the space $N$ can be represented as the space of solutions of the system of linear equations $L v=0$, where $v$ is the column vector of coefficients of polynomials $\alpha_{\gamma}, \gamma \in T_{0}^{0}$. Here, we do not describe the structure of the matrix $L$ in the general case because it is simpler to understand it from the examples below.

We simply note that the number of columns in matrix $L$ is $(m-r)\left(m^{\prime}-r^{\prime}\right) n$ and the number of rows is $(m+$ $1)\left(m^{\prime}-r^{\prime}\right) l_{h}+\left(m^{\prime}+1\right)(m-r) l_{v}$. One can compute $\operatorname{dim} N$ as

$$
\operatorname{dim} N=(m-r)\left(m^{\prime}-r^{\prime}\right) n-\operatorname{rank} L .
$$

Thus, for some T-mesh T,

$$
\begin{gathered}
h_{m, m^{\prime}}^{r, r^{\prime}}(T)=(m+1)\left(m^{\prime}-r^{\prime}\right) l_{h}+ \\
\quad\left(m^{\prime}+1\right)(m-r) l_{v}-\operatorname{rank} L .
\end{gathered}
$$

\section{Example of T-mesh with instability in the dimen- sion of a spline space $S(3,3,2,2)(T)$}

In order to establish the instability in the dimension of a spline space, it is necessary to find a T-mesh in which the dimension jumps for some small change in the mesh. The proof of dimension jumping is based on calculating the rank of the corresponding matrix $L$. For $m=m^{\prime}, r=r^{\prime}=m-1$, examples of such T-meshes are found in [1].

We describe in detail the example given in [1] for the case $m=m^{\prime}=3, r=r^{\prime}=2$.

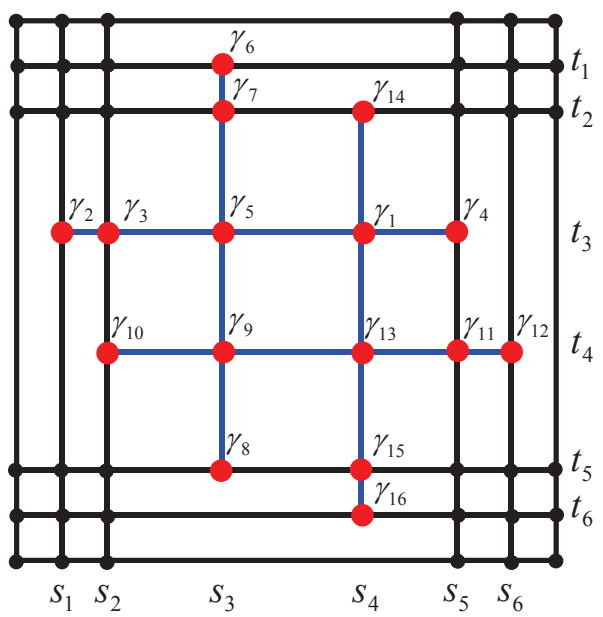

Figure 3: Unstable T-mesh for $m=m^{\prime}=3, r=r^{\prime}=2$.

For the T-mesh shown in Fig. 3, every element of the space $N$ (see (3),(4)) is simply the 16-th dimensional column vector $v$ of the real numbers $\alpha_{j}, j=1 \ldots 16$ (each $\alpha_{j}$ corresponds to the vertex $\gamma_{j}, j=1 \ldots 16$ ), such that the following equations hold for arbitrary $x$ and $y$ :

$$
\begin{gathered}
\sum_{i=1}^{5} \alpha_{i}\left(x-x_{\gamma_{i}}\right)^{3}=0, \\
\Sigma_{i=5}^{9} \alpha_{i}\left(y-y_{\gamma_{i}}\right)^{3}=0, \\
\sum_{i=9}^{13} \alpha_{i}\left(x-x_{\gamma_{i}}\right)^{3}=0, \\
\sum_{i=13}^{16} \alpha_{i}\left(y-y_{\gamma_{i}}\right)^{3}+\alpha_{1}\left(y-y_{\gamma_{1}}\right)^{3}=0 .
\end{gathered}
$$

Note that these equations correspond to the maximal interior segments $\gamma_{2} \gamma_{4}, \gamma_{6} \gamma_{8}, \gamma_{10} \gamma_{12}$, and $\gamma_{14} \gamma_{16}$, respectively.

Because the coefficients of the polynomials in these equations are zero, one can obtain the following system of linear equations:

$$
\alpha_{2} s_{1}^{k}+\alpha_{3} s_{2}^{k}+\alpha_{5} s_{3}^{k}+\alpha_{1} s_{4}^{k}+\alpha_{4} s_{5}^{k}=0
$$




$$
\begin{gathered}
\alpha_{6} t_{1}^{k}+\alpha_{7} t_{2}^{k}+\alpha_{5} t_{3}^{k}+\alpha_{9} t_{4}^{k}+\alpha_{8} t_{5}^{k}=0, \\
\alpha_{10} s_{2}^{k}+\alpha_{9} s_{3}^{k}+\alpha_{13} s_{4}^{k}+\alpha_{11} s_{5}^{k}+\alpha_{12} s_{6}^{k}=0, \\
\alpha_{14} t_{2}^{k}+\alpha_{1} t_{3}^{k}+\alpha_{13} t_{4}^{k}+\alpha_{15} t_{5}^{k}+\alpha_{16} t_{6}^{k}=0,
\end{gathered}
$$

for $k=0 \ldots 3$.

By writing these linear equations in the form $L v=0$, we obtain the following form for the $16 \times 16$ matrix $L$ :

$$
\left(\begin{array}{ccccccccc}
1 & 1 & 1 & 1 & 1 & 0 & 0 & 0 & \\
s_{4} & s_{1} & s_{2} & s_{5} & s_{3} & 0 & 0 & 0 & \\
s_{4}^{2} & s_{1}^{2} & s_{2}^{2} & s_{5}^{2} & s_{3}^{2} & 0 & 0 & 0 & \\
s_{4}^{3} & s_{1}^{3} & s_{2}^{3} & s_{5}^{3} & s_{3}^{3} & 0 & 0 & 0 & \\
0 & 0 & 0 & 0 & 1 & 1 & 1 & 1 & \\
0 & 0 & 0 & 0 & t_{3} & t_{1} & t_{2} & t_{5} & \\
0 & 0 & 0 & 0 & t_{3}^{2} & t_{1}^{2} & t_{2}^{2} & t_{5}^{2} & \\
0 & 0 & 0 & 0 & t_{3}^{3} & t_{1}^{3} & t_{2}^{3} & t_{5}^{3} & \\
0 & 0 & 0 & 0 & 0 & 0 & 0 & 0 & \\
0 & 0 & 0 & 0 & 0 & 0 & 0 & 0 & \\
0 & 0 & 0 & 0 & 0 & 0 & 0 & 0 & \\
0 & 0 & 0 & 0 & 0 & 0 & 0 & 0 & \\
1 & 0 & 0 & 0 & 0 & 0 & 0 & 0 & \\
t_{3} & 0 & 0 & 0 & 0 & 0 & 0 & 0 & \\
t_{3}^{2} & 0 & 0 & 0 & 0 & 0 & 0 & 0 & \\
t_{3}^{3} & 0 & 0 & 0 & 0 & 0 & 0 & 0 & \\
& 0 & 0 & 0 & 0 & 0 & 0 & 0 & 0 \\
& 0 & 0 & 0 & 0 & 0 & 0 & 0 & 0 \\
& 0 & 0 & 0 & 0 & 0 & 0 & 0 & 0 \\
& 0 & 0 & 0 & 0 & 0 & 0 & 0 & 0 \\
& 1 & 0 & 0 & 0 & 0 & 0 & 0 & 0 \\
& t_{4} & 0 & 0 & 0 & 0 & 0 & 0 & 0 \\
& t_{4}^{2} & 0 & 0 & 0 & 0 & 0 & 0 & 0 \\
& t_{4}^{3} & 0 & 0 & 0 & 0 & 0 & 0 & 0 \\
& 1 & 1 & 1 & 1 & 1 & 0 & 0 & 0 \\
& s_{3} & s_{2} & s_{5} & s_{6} & s_{4} & 0 & 0 & 0 \\
& s_{3}^{2} & s_{2}^{2} & s_{5}^{2} & s_{6}^{2} & s_{4}^{2} & 0 & 0 & 0 \\
& s_{3}^{3} & s_{2}^{3} & s_{5}^{3} & s_{6}^{3} & s_{4}^{3} & 0 & 0 & 0 \\
& 0 & 0 & 0 & 0 & 1 & 1 & 1 & 1 \\
& 0 & 0 & 0 & 0 & t_{4} & t_{2} & t_{5} & t_{6} \\
& 0 & 0 & 0 & 0 & t_{4}^{2} & t_{2}^{2} & t_{5}^{2} & t_{6}^{2} \\
& 0 & 0 & 0 & 0 & t_{4}^{3} & t_{2}^{3} & t_{5}^{3} & t_{6}^{3}
\end{array}\right)
$$

It is shown in [1] that the determinant of the matrix $L$ is zero if and only if

$$
\frac{\left(s_{3}-s_{1}\right)\left(s_{6}-s_{4}\right)}{\left(t_{3}-t_{1}\right)\left(t_{6}-t_{4}\right)}=\frac{\left(s_{4}-s_{1}\right)\left(s_{6}-s_{3}\right)}{\left(t_{4}-t_{1}\right)\left(t_{6}-t_{3}\right)} .
$$

This equation holds, for instance, when $s_{i}=t_{i}, i=$ $1 \ldots 6$. However, a small change in coordinates $s_{i}, t_{i}, i=$ $1 \ldots 6$ makes the matrix $L$ non-degenerate, which implies jumping of the $\operatorname{rank}(L)$, and therefore, because of (2) and (5) jumping of the dimension of the spline space. The bound of [11] yields in this case $0 \leq h_{3,3}^{2,2}(T) \leq 1$.
Note that the structure of the T-meshes in [1] is not complicated, and these T-meshes only have four maximal interior segments.

The observation of instability in the dimension of $S(m, m, m-1, m-1)(T)$, given in [1], inspired us to construct examples of T-meshes with instability in the dimension for the cases $S(4,4,2,2)(T)$ and $S(5,5,3,3)(T)$.

\section{Examples of T-meshes with instability in the dimension of spline spaces $S(4,4,2,2)(T)$ and $S(5,5,3,3)(T)$}

In this section, we describe certain types of T-meshes $T$ and prove the instability in the dimension of spline spaces $S(4,4,2,2)(T)$ and $S(5,5,3,3)(T)$.

We start with the case $S(5,5,3,3)(T)$, because for the example of the T-mesh given for $S(2,2,1,1)(T)[1]$, instability in the dimension of the space $S(5,5,3,3)(T)$ has been demonstrated. This T-mesh is shown in Fig. 4.

\subsection{Case: $m=m^{\prime}=5, r=r^{\prime}=3$}

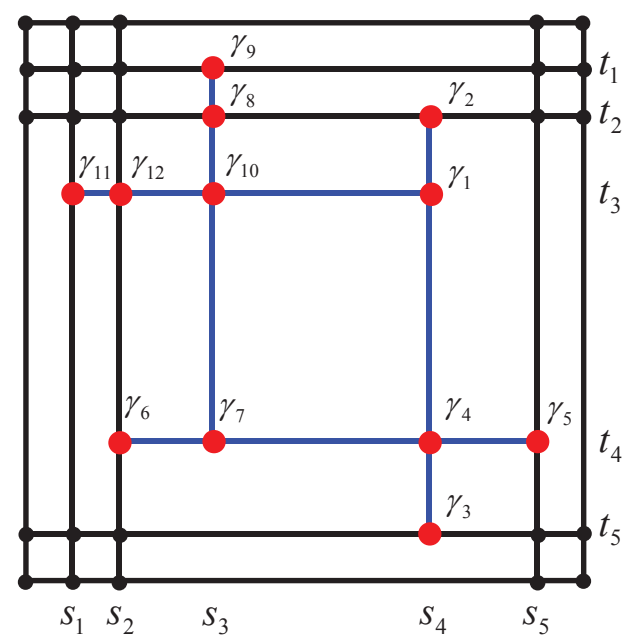

Figure 4: Unstable T-mesh for cases $m=m^{\prime}=5, r=r^{\prime}=3$ and $m=m^{\prime}=2, r=r^{\prime}=1$.

The polynomials $\alpha_{\gamma_{i}} \in R_{(1,1)}, \gamma_{i} \in T_{0}^{0}, i=1 \ldots 12$ can be represented as

$$
\begin{gathered}
\alpha_{\gamma_{i}}=k_{1 i}\left(x-x_{\gamma_{i}}\right)\left(y-y_{\gamma_{i}}\right)+k_{2 i}\left(x-x_{\gamma_{i}}\right) \\
+k_{3 i}\left(y-y_{\gamma_{i}}\right)+k_{4 i} .
\end{gathered}
$$

Let $v$ denote the column vector of the length 48 , such that for $j=4 l+r, r=j \bmod 4$, the $j$-th coordinate of $v$ equals $k_{(r+1)(l+1)}$. Then, the set of polynomials

$$
\left\langle\alpha_{\gamma_{i}}, i=1 \ldots 12\right\rangle
$$


is in $N$ (see (3) and (4)) if and only if the following polynomials are zero:

$$
\begin{aligned}
& \Sigma_{\gamma_{i} \in \rho} k_{1 i}\left(x-x_{\gamma_{i}}\right)^{5}+k_{3 i}\left(x-x_{\gamma_{i}}\right)^{4}=0, \\
& \Sigma_{\gamma_{i} \in \rho} k_{2 i}\left(x-x_{\gamma_{i}}\right)^{5}+k_{4 i}\left(x-x_{\gamma_{i}}\right)^{4}=0
\end{aligned}
$$

if $\rho$ is the maximal horizontal interior segment, and

$$
\begin{gathered}
\Sigma_{\gamma_{i} \in \rho} k_{1 i}\left(y-y_{\gamma_{i}}\right)^{5}+k_{2 i}\left(y-y_{\gamma_{i}}\right)^{4}=0 \\
\Sigma_{\gamma_{i} \in \rho} k_{3 i}\left(y-y_{\gamma_{i}}\right)^{5}+k_{4 i}\left(y-y_{\gamma_{i}}\right)^{4}=0
\end{gathered}
$$

if $\rho$ is the maximal vertical interior segment.

Let $\mathbf{V}_{t}$ denote, for some given $t$, the rectangular $12 \times 4$ matrix of the form

$$
\mathbf{V}_{t}=\left(\begin{array}{cccc}
1 & 0 & 0 & 0 \\
t & -\frac{1}{5} & 0 & 0 \\
t^{2} & -\frac{2}{5} t & 0 & 0 \\
t^{3} & -\frac{3}{5} t^{2} & 0 & 0 \\
t^{4} & -\frac{4}{5} t^{3} & 0 & 0 \\
t^{5} & -t^{4} & 0 & 0 \\
0 & 0 & 1 & 0 \\
0 & 0 & t & -\frac{1}{5} \\
0 & 0 & t^{2} & -\frac{2}{5} t \\
0 & 0 & t^{3} & -\frac{3}{5} t^{2} \\
0 & 0 & t^{4} & -\frac{4}{5} t^{3} \\
0 & 0 & t^{5} & -t^{4}
\end{array}\right),
$$

and $\mathbf{H}_{s}$ be a matrix of the form

$$
\mathbf{H}_{s}=\left(\begin{array}{cccc}
1 & 0 & 0 & 0 \\
s & 0 & -\frac{1}{5} & 0 \\
s^{2} & 0 & -\frac{2}{5} s & 0 \\
s^{3} & 0 & -\frac{3}{5} s^{2} & 0 \\
s^{4} & 0 & -\frac{4}{5} s^{3} & 0 \\
s^{5} & 0 & -s^{4} & 0 \\
0 & 1 & 0 & 0 \\
0 & s & 0 & -\frac{1}{5} \\
0 & s^{2} & 0 & -\frac{2}{5} s \\
0 & s^{3} & 0 & -\frac{3}{5} s^{2} \\
0 & s^{4} & 0 & -\frac{4}{5} s^{3} \\
0 & s^{5} & 0 & -s^{4}
\end{array}\right) .
$$

Then, from equations (6) and (7), one can obtain the system of linear equations for coefficients $k_{j i}, j=$ $1 \ldots 4$, and $i=1 \ldots 12$ in the following manner:

$$
\Sigma_{\gamma_{i} \in \rho} \mathbf{H}_{x_{\gamma_{i}}}\left(\begin{array}{c}
k_{1 i} \\
k_{2 i} \\
k_{3 i} \\
k_{4 i}
\end{array}\right)=0
$$

if $\rho$ is the maximal horizontal interior segment, and

$$
\Sigma_{\gamma_{i} \in \rho} \mathbf{V}_{y_{\gamma_{i}}}\left(\begin{array}{c}
k_{1 i} \\
k_{2 i} \\
k_{3 i} \\
k_{4 i}
\end{array}\right)=0
$$

if $\rho$ is the maximal vertical interior segment.

We consider the system of linear equations (8) and (9) for coefficients $k_{j i}, j=1 \ldots 4$, and $i=1 \ldots 12$ in terms of the linear system $L v=0$. Then, the matrix $L$ has $4 \times 12=48$ columns and $12 \times 4=48$ rows. By using equations (8) and (9) and sequentially taking the maximal interior segments $\gamma_{2} \gamma_{3}, \gamma_{5} \gamma_{6}, \gamma_{7} \gamma_{9}$, and $\gamma_{1} \gamma_{11}$ (see Fig. 4), one can represent the matrix $L$ in terms of $12 \times 4$ blocks $\mathbf{H}_{x_{\gamma_{i}}}, \mathbf{V}_{y_{\gamma_{i}}}, i=1 \ldots 12$ and zero blocks $\mathbf{0}$ filled with nulls in the following manner:

$$
\left(\begin{array}{ccccccc}
\mathbf{V}_{t_{3}} & \mathbf{V}_{t_{2}} & \mathbf{V}_{t_{5}} & \mathbf{V}_{t_{4}} & \mathbf{0} & \mathbf{0} & \\
\mathbf{0} & \mathbf{0} & \mathbf{0} & \mathbf{H}_{s_{4}} & \mathbf{H}_{s_{5}} & \mathbf{H}_{s_{2}} & \\
\mathbf{0} & \mathbf{0} & \mathbf{0} & \mathbf{0} & \mathbf{0} & \mathbf{0} & \\
\mathbf{H}_{s_{4}} & \mathbf{0} & \mathbf{0} & \mathbf{0} & \mathbf{0} & \mathbf{0} & \\
& \mathbf{0} & \mathbf{0} & \mathbf{0} & \mathbf{0} & \mathbf{0} & \mathbf{0} \\
& \mathbf{H}_{s_{3}} & \mathbf{0} & \mathbf{0} & \mathbf{0} & \mathbf{0} & \mathbf{0} \\
& \mathbf{V}_{t_{4}} & \mathbf{V}_{t_{2}} & \mathbf{V}_{t_{1}} & \mathbf{V}_{t_{3}} & \mathbf{0} & \mathbf{0} \\
& \mathbf{0} & \mathbf{0} & \mathbf{0} & \mathbf{H}_{s_{3}} & \mathbf{H}_{s_{1}} & \mathbf{H}_{s_{2}}
\end{array}\right)
$$

Thus, according to (5), for the given T-mesh, $h_{5,5}^{3,3}$ equals $48-\operatorname{rank}(L)$.

In this work, we do not consider the question of calculating the rank of $L$ for an arbitrary set of values $t_{1}, \ldots, t_{5}$ and $s_{1}, \ldots, s_{5}$ to describe all possible positions with instability in the dimension.

We are simply interested in the topology of the $\mathrm{T}$-mesh and in providing some concrete values for $t_{1}, \ldots, t_{5}$ and $s_{1}, \ldots, s_{5}$ such that dimension jumping occurs for some small change in these values.

For instance, let us consider the following values:

$$
\begin{gathered}
t_{1}=s_{1}=-2, t_{2}=s_{2}=-1, t_{3}=s_{3}=0, \\
s_{4}=t_{4}=1, s_{5}=t_{5}=2 .
\end{gathered}
$$

Then, $\operatorname{rank} L=46$. In order to be both rigorous and simple, we fix all values except $t_{1}$ and calculate the determinant of the matrix $L$. One can check that the determinant is nonzero when $t_{1} \neq-2$, implying that the rank of $L$ is equal to 48 .

Therefore, the dimension of $S(5,5,3,3)$ is increased by 2 when $t_{1}=-2$, and therefore, we have dimension jumping.

Notice that the bound of [11] yields $0 \leq h_{5,5}^{3,3}(T) \leq 4$.

4.2. Case: $m=m^{\prime}=4, r=r^{\prime}=2$.

For $S(4,4,2,2)(T)$, the example is a bit more curious. It is shown in Fig. 5.

The approach used in this subsection is the same as that used in the previous one. 


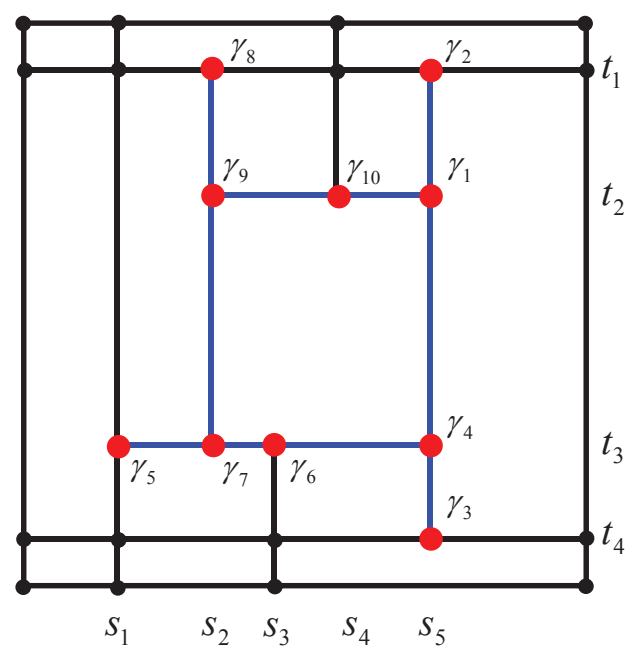

Figure 5: Unstable T-mesh for $m=m^{\prime}=4, r=r^{\prime}=2$

Again, the polynomials $\alpha_{\gamma_{i}} \in R_{(1,1)}, \gamma_{i} \in T_{0}^{0}, i=$ $1 \ldots 10$ can be represented as

$\alpha_{\gamma_{i}}=k_{1 i}\left(x-x_{\gamma_{i}}\right)\left(y-y_{\gamma_{i}}\right)+k_{2 i}\left(x-x_{\gamma_{i}}\right)+k_{3 i}\left(y-y_{\gamma_{i}}\right)+k_{4 i}$.

Let $v$ denote the column vector of length 40, such that for $j=4 l+r, r=j \bmod 4$, the $j$-th coordinate of $v$ equals $k_{(r+1)(l+1)}$. Then, the set of polynomials

$$
\left\langle\alpha_{\gamma_{i}}, i=1 \ldots 10\right\rangle
$$

is in space $N$ (see (3) and (4)) if and only if the following polynomials are zero:

$$
\begin{gathered}
\Sigma_{\gamma_{i} \in \rho} k_{1 i}\left(x-x_{\gamma_{i}}\right)^{4}+k_{3 i}\left(x-x_{\gamma_{i}}\right)^{3}=0, \\
\Sigma_{\gamma_{i} \in \rho} k_{2 i}\left(x-x_{\gamma_{i}}\right)^{4}+k_{4 i}\left(x-x_{\gamma_{i}}\right)^{3}=0
\end{gathered}
$$

if $\rho$ is the maximal horizontal interior segment, and

$$
\begin{gathered}
\Sigma_{\gamma_{i} \in \rho} k_{1 i}\left(y-y_{\gamma_{i}}\right)^{4}+k_{2 i}\left(y-y_{\gamma_{i}}\right)^{3}=0, \\
\Sigma_{\gamma_{i} \in \rho} k_{3 i}\left(y-y_{\gamma_{i}}\right)^{4}+k_{4 i}\left(y-y_{\gamma_{i}}\right)^{3}=0
\end{gathered}
$$

if $\rho$ is the maximal vertical interior segment.

We define the $10 \times 4$ matrices $\mathbf{V}_{t}$ and $\mathbf{H}_{s}$ as follows:

$$
\mathbf{V}_{t}=\left(\begin{array}{cccc}
1 & 0 & 0 & 0 \\
t & -\frac{1}{4} & 0 & 0 \\
t^{2} & -\frac{1}{2} t & 0 & 0 \\
t^{3} & -\frac{3}{4} t^{2} & 0 & 0 \\
t^{4} & -t^{3} & 0 & 0 \\
0 & 0 & 1 & 0 \\
0 & 0 & t & -\frac{1}{4} \\
0 & 0 & t^{2} & -\frac{1}{2} t \\
0 & 0 & t^{3} & -\frac{3}{4} t^{2} \\
0 & 0 & t^{4} & -t^{3}
\end{array}\right),
$$

$$
\mathbf{H}_{s}=\left(\begin{array}{cccc}
1 & 0 & 0 & 0 \\
s & 0 & -\frac{1}{4} & 0 \\
s^{2} & 0 & -\frac{1}{2} s & 0 \\
s^{3} & 0 & -\frac{3}{4} s^{2} & 0 \\
s^{4} & 0 & -s^{3} & 0 \\
0 & 1 & 0 & 0 \\
0 & s & 0 & -\frac{1}{4} \\
0 & s^{2} & 0 & -\frac{1}{2} s \\
0 & s^{3} & 0 & -\frac{3}{4} s^{2} \\
0 & s^{4} & 0 & -s^{3}
\end{array}\right) .
$$

Then, from equations (10) and (11), one can obtain the system of linear equations for coefficients $k_{j i}, j=$ $1 \ldots 4$, and $i=1 \ldots 10$ in the following manner:

$$
\Sigma_{\gamma_{i} \in \rho} \mathbf{H}_{x_{\gamma_{i}}}\left(\begin{array}{c}
k_{1 i} \\
k_{2 i} \\
k_{3 i} \\
k_{4 i}
\end{array}\right)=0
$$

if $\rho$ is the maximal horizontal interior segment, and

$$
\Sigma_{\gamma_{i} \in \rho} \mathbf{V}_{y_{\gamma_{i}}}\left(\begin{array}{c}
k_{1 i} \\
k_{2 i} \\
k_{3 i} \\
k_{4 i}
\end{array}\right)=0
$$

if $\rho$ is the maximal vertical interior segment.

We consider the system of linear equations (12) and (13) for coefficients $k_{j i}, j=1 \ldots 4$, and $i=1 \ldots 10$ in terms of the linear system $L v=0$. Then, the matrix $L$ has $4 \times 10=40$ columns and $10 \times 4=40$ rows. By using equations (12) and (13) and sequentially taking maximal interior segments $\gamma_{2} \gamma_{3}, \gamma_{4} \gamma_{5}, \gamma_{7} \gamma_{8}$, and $\gamma_{1} \gamma_{9}$ (see Fig. 5), one can represent the matrix $L$ in terms of $10 \times 4$ blocks $\mathbf{H}_{x_{\gamma_{i}}}, \mathbf{V}_{y_{\gamma_{i}}}, i=1 \ldots 10$ and zero blocks $\mathbf{0}$ filled with nulls in the following manner:

$$
\begin{aligned}
& \left(\begin{array}{ccccc}
\mathbf{V}_{t_{2}} & \mathbf{V}_{t_{1}} & \mathbf{V}_{t_{4}} & \mathbf{V}_{t_{3}} & \mathbf{0} \\
\mathbf{0} & \mathbf{0} & \mathbf{0} & \mathbf{H}_{s_{5}} & \mathbf{H}_{s_{1}} \\
\mathbf{0} & \mathbf{0} & \mathbf{0} & \mathbf{0} & \mathbf{0} \\
\mathbf{H}_{s_{5}} & \mathbf{0} & \mathbf{0} & \mathbf{0} & \mathbf{0}
\end{array}\right. \\
& \left.\begin{array}{ccccc}
\mathbf{0} & \mathbf{0} & \mathbf{0} & \mathbf{0} & \mathbf{0} \\
\mathbf{H}_{s_{3}} & \mathbf{H}_{s_{2}} & \mathbf{0} & \mathbf{0} & \mathbf{0} \\
\mathbf{0} & \mathbf{V}_{t_{3}} & \mathbf{V}_{t_{1}} & \mathbf{V}_{t_{2}} & \mathbf{0} \\
\mathbf{0} & \mathbf{0} & \mathbf{0} & \mathbf{H}_{s_{2}} & \mathbf{H}_{s_{4}}
\end{array}\right)
\end{aligned}
$$

We take the following values for $t_{1}, t_{2}, t_{3}, t_{4}$ and $s_{1}, s_{2}, s_{5}$ :

$$
\begin{gathered}
t_{1}=0, t_{2}=1, t_{3}=3, t_{4}=4, \\
s_{1}=0, s_{2}=1, s_{5}=3 .
\end{gathered}
$$


If we substitute $s_{3}=s_{4}$, then the rank of $L$ is 38 , otherwise it is 39 . This means that the dimension of $S(4,4,2,2)$ for the given T-mesh increases by 1 when $s_{3}=s_{4}$. Again, the bound of [11] yields $0 \leq h_{4,4}^{2,2}(T) \leq$ 4.

Note that both examples have only four maximal interior segments and their structures are quite simple.

\section{Conclusions and future work}

In this work, we proved the instability in the dimension of a spline space $S\left(m, m^{\prime}, r, r^{\prime}\right)(T)$ over certain types of T-meshes $T$ for the new cases $m=m^{\prime}=4, r=$ $r^{\prime}=2$ and $m=m^{\prime}=5, r=r^{\prime}=3$. The constructed examples have only four maximal interior segments and appear to be the simplest ones in some sense. One can construct more complicated examples of T-meshes with instability in the dimension by some combinations of the T-meshes shown in Figs. 4 and 5 for the cases $m=m^{\prime}=5, r=r^{\prime}=3$ and $m=m^{\prime}=4, r=r^{\prime}=2$, respectively.

To prove the instability in the dimension, we specified the values of the node coordinates of the T-mesh for the following two reasons. First, our main focus is on understanding the topology of a T-mesh with instability in the dimension, and second, calculating the rank of the matrix $L$ is simplified considerably. This work does not accurately analyze the algebraic conditions for the node coordinates for the dimension to be unstable. However, such an analysis could be carried out by implementing a row reduction procedure to calculate the rank of matrix $L$.

In the future, the following problems would be interesting to explore. The first is the investigation of the instability in the dimension for the following, more general cases: $m=m^{\prime}, r=r^{\prime}$ and $2 r+1>m$. The problem is that the analysis of the rank of matrix $L$ is nontrivial in more general cases; therefore, we might need to use another interpretation of the dimension formula. The second is the description of classes of T-meshes for the dimension of a space $S\left(m, m^{\prime}, r, r^{\prime}\right)(T)$ to be stable and controlled by some topological characteristics. The work of [11] made progress in this regard, but we believe that it could be considerably strengthened by a more careful analysis of $h_{m, m^{\prime}}^{r, r^{\prime}}$. We intend to investigate these questions in our future work.

\section{Acknowledgement}

This research was supported by the National Research Foundation of Korea(NRF) grant funded by the
Korea government(MEST) (No. 2011-0018023) and partially supported by the Industrial Strategic technology development program, 10035474, Development of inspection platform technology based on 3-dimensional $\mathrm{X}$-ray images funded by the Ministry of Knowledge Economy(MKE, Korea).

\section{References}

[1] X. Li, F. Chen, On the instability in the dimension of splines spaces over T-meshes, Computer Aided Geometric Design 28 (2011) 420-426.

[2] J. Deng, F. Chen, Y. Feng, Dimension of spline spaces over T-meshes, Journal of Computational and Applied Mathematics 194 (2006) 267-283.

[3] J. Deng, F. Chen, X. Li, C. Hu, W. Tong, Z. Yang, Y. Feng, Polynomial splines over hierarchical T-meshes, Graphical models 74 (2008) 76-86

[4] X. Li, J. Deng, F. Chen, Surface modeling with polynomial splines over hierarchical T-meshes, Visual Computer 23 (2007) 1027-1033.

[5] J. A. Cottrell, T. J. R. Hughes, Y. Bazilevs, Isogeometric Analysis: Toward Integration of CAD and FEA, John Wiley \& Sons, 2009.

[6] N. Nguyen-Thanh, H. Nguyen-Xuan, S. Bordas, T. Rabczuk, Isogeometric analysis using polynomial splines over hierarchical T-meshes for two-dimensional elastic solids, Computer Methods in Applied Mechanics and Engineering 200 (2011) 1892-1908.

[7] A.-V. Vuong, C. Giannelli, B. Jüttler, B. Simeon, A hierarchical approach to adaptive local refinement in isogeometric analysis, Computer Methods in Applied Mechanics and Engineering 200 (2011) 3554-3567.

[8] X. Li, J. Deng, F. Chen, Polynomial splines over general Tmeshes, The Visual Computer 26 (4) (2010) 277-286.

[9] Z. Huang, J. Deng, Y. Feng, F. Chen, New proof of dimension formula of spline spaces over T-meshes via smoothing cofactors, Journal of Computational Mathematics 24 (4) (2006) 501514.

[10] C.-J. Li, R.-H. Wang, F. Zhang, Improvement on the dimension of spline spaces on T-mesh, Journal of Information and Computational Science 3 (2) (2006) 235-244.

[11] B. Mourrain, On the dimension of spline spaces on planar T-subdivisions, preprint, inria-00533187, available at http://arxiv.org/abs/1011.1752v1. 